\title{
Vantaggi dell'utilizzo della tecnica Buttonhole. Studio di un caso clinico in un Centro Dialisi in Puglia
}

\author{
Patrizia Quaranta ${ }^{1}$, Luigi Nuccio ${ }^{2}$, Marianeve Alessio ${ }^{1}$, Anna Maria Alfarano ${ }^{1}$, Ambrogio Carluccio $^{1}$, Loredana Melcarne ${ }^{1}$, \\ Maria Rosaria Nuccio ${ }^{1}$, Tonia Quarta ${ }^{1}$, Antonella Schirinzi ${ }^{1}$, Vitale Nuzzo ${ }^{3}$ \\ ${ }^{1}$ Infermiere U.O. Nefrologia e Dialisi Ospedale "Card. G. Panico", Tricase (LE) \\ ${ }^{2}$ Infermiere Coordinatore U.O. Nefrologia e Dialisi Ospedale "Card. G. Panico", Tricase (LE) \\ ${ }^{3}$ Medico Responsabile U.O. Nefrologia e Dialisi Ospedale "Card. G. Panico", Tricase (LE)
}

\section{The advantages of the buttonhole technique. A case report in a dialysis center in Puglia}

Native AVF represents the best vascular access for patients undergoing chronic hemodialysis treatments. Accordingly to KDOQI 2006, constant site cannulation technique (or butthonhole, $\mathrm{BH}$ ) can reduce infiltration events, cannulation pain, make self cannulation process easier and maintain a better vein outflow integrity. Nursing staff need to be trained in order to acquire and improve AV cannulation skills.

The objective of this study is to demonstrate benefits of buttonhole technique for AVF cannulations in chronic hemodialysis patients by a case report experience in our centre.

Based on our experience, only if adequate training will be provided to nursing staff and greater emphasis will be given to patients empowerment and $\mathrm{AV}$ self management, $\mathrm{BH}$ technique could bring greater advantages and less complications for both patients and staff.

$\mathrm{BH}$ technique, if properly applied, can reduce infiltration risk, bleeding and pain while preserving vein physiology. Keywords: Buttonhole puncture method, Hemodialysis, Vascular access

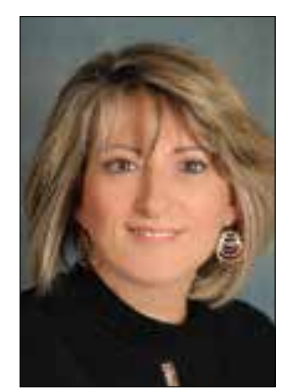

Patrizia Quaranta

\section{Introduzione}

La fistola arterovenosa (FAV) allestita con vasi nativi rappresenta il migliore accesso vascolare per i pazienti in emodialisi cronica. Al fine di garantire la sopravvivenza e di migliorare la qualità di vita degli stessi pazienti, un adeguato accesso vascolare deve rispondere a determinate caratteristiche: 1 ) avere una posizione e una pungibilità facili, 2) fornire un flusso di sangue sufficiente per ottenere una dialisi efficace, 3) presentare una lunga pervietà e 4) assicurare un basso tasso di complicanze (1). Diverse tecniche di puntura della FAV sono state utilizzate negli anni: la scala di corda

Accepted: January 22, 2016

Published online: February 17, 2016

Indirizzo per la corrispondenza:

Dr.ssa Patrizia Quaranta

Infermiera di Emodialisi

Ospedale "Card. G. Panico"

Via S. Pio $X, 4$

73039 Tricase (LE)

patriziaq66@gmail.com
(Rope-Ladder) con punture lungo il decorso del vaso, tecnica più frequentemente utilizzata in Europa e negli Stati membri (2), la tecnica ad area (area puncture), con punture concentrate in piccole porzioni del vaso, che può più facilmente creare danni e sviluppare stenosi e aneurismi (3), e, infine, la tecnica a sito costante o ad asola o a occhiello (constant-site), in cui gli aghi sono inseriti esattamente nello stesso punto ad ogni seduta di dialisi. La tecnica a sito costante, introdotta agli inizi degli anni ' 70 da Zbylut J. Twardowski, Professore Emerito dell'University of Missouri School of Medicine, Columbia (4), è stata descritta per la prima volta nella letteratura polacca nel 1977 e, due anni dopo, sul giornale Dialysis \& Transplantation (D. \& T.). Tale metodo, che è stato successivamente rinominato "Buttonhole Puncture" da G. Kronung (5), ha la peculiarità di utilizzare punture ripetute nello stesso sito, riducendo il rischio di dilatazioni aneurismatiche e/o di stenosi. Viene utilizzato più frequentemente da pazienti sottoposti a dialisi domiciliare, perché ha la caratteristica di essere facilmente applicabile per l'auto-puntura ("self cannulation") e ben si addice a essere applicato da un unico puntore ("single sticker"). Questa pratica non è stata molto presa in considerazione per diversi motivi (mancanza di aghi smussi, tipologia di fistola, dettagli della tecnica o ricorso a "multiple stickers" durante la preparazione del sito). Di certo, dalla pubblicazione di Twardowski sulla rivista D. \& T. del 1995 a oggi, sembra che si stia diffondendo a "mac- 
chia d'olio". Molti ricercatori stanno lavorando sul metodo BH e numerosi studi sono in corso di pubblicazione per dimostrare I'utilità in termini di benefici e di una migliore conservazione dell'accesso vascolare (4). Non sono ancora disponibili evidenze scientifiche sull'applicazione della tecnica per i pazienti con protesi ("graft"), soprattutto per quelle in Politetrafluoroetilene (PTFE) o simili (6).

È importante che, durante il periodo di preparazione del sito, la fistola venga punta dallo stesso operatore esperto ("single sticker") con aghi taglienti per almeno 8-12 sedute di dialisi (4). Per agevolare e accelerare la creazione del percorso sottocutaneo, è stato creato un piolo in policarbonato lungo $5 \mathrm{~mm}$ da applicare nello stesso sito di puntura, dopo l'emostasi (6). Tale dispositivo viene mantenuto fino all'inserimento di un nuovo ago per la successiva seduta emodialitica. Questa manovra viene ripetuta per un periodo di 2 settimane fino a quando non si è definito il percorso dell'ago nel sottocute. L'operatore, nell'inserzione degli aghi, deve usare sempre le stesse direzione, angolazione e profondità di penetrazione (7). Successivamente, si passa all'utilizzo di aghi smussi e, solo dopo aver creato dei buoni siti, si può estendere a tutto il personale la puntura della fistola (8). Una peculiarità degli aghi smussi consiste nel fatto che questi, se correttamente inseriti, tendono a creare un sentiero ben stabilito senza tagliare i tessuti adiacenti, inconveniente che potrebbe provocare sanguinamento del sito di venipuntura, formazione di ematomi e aumento del tempo di emostasi.

Secondo le Linee Guida KDOQI del 2006 (Clinical Practice Recommendations for Guideline 3: cannulation of fistulae and grafts and accession of dialysis catheters and ports) (9), è sempre più evidente che la puntura a sito costante comporta meno rischi di infiltrazioni, può essere meno dolorosa, può contribuire a preservare l'integrità del vaso e può essere più facile da reperire per i pazienti che si auto-pungono. In più, una riduzione dei problemi legati all'accesso vascolare si traduce in una diminuzione di interventi diagnostici e terapeutici e, di conseguenza, in una riduzione delle degenze e dei costi (10). Secondo le stesse Linee Guida, tutti i pazienti con FAV possono essere candidati alla puntura a sito costante e tutti dovrebbero essere educati alla cura della stessa e incoraggiati all'auto-puntura, purché ne siano capaci e abbiano seguito un'adeguata formazione. L'auto-puntura ha una serie di vantaggi:

1. I'autogestione consente un maggiore controllo della malattia e del trattamento: i pazienti vengono messi nella condizione di occuparsi maggiormente di loro stessi e di avere meno paura della dialisi, del dolore e dei problemi legati all'accesso vascolare (10);

2. i pazienti sono abili nel sentire l'ago all'interno del vaso durante la procedura e nell'avvertire precocemente eventuali problemi;

3. sono più stimolati ad allontanarsi dal Centro Dialisi di ap- partenenza, anche se per periodi di vacanza, superando molte difficoltà derivanti da cambiamenti di abitudini legati alla cronicità della terapia dialitica (11).

\section{Obiettivo}

L'obiettivo di questo studio è di dimostrare i benefici dell'utilizzo della tecnica $\mathrm{BH}$ per la puntura delle FAV native nei pazienti emodializzati cronici, attraverso l'esperienza del nostro Centro e la descrizione di un caso clinico.

\section{L'esperienza del Centro Dialisi "Santa Marcellina" di S. Maria di Leuca}

Il Centro ad Assistenza Decentrata (CAD) è una struttura specialistica finalizzata a garantire la dialisi a pazienti con instabilità clinica ma gestibile grazie alla presenza continuativa del nefrologo durante tutta la seduta dialitica. La struttura "Santa Marcellina" nasce nel 2004 per garantire il trattamento dialitico a pazienti provenienti dalle zone limitrofe e dare ospitalità a quelle persone emodializzate che vengono nel Salento per diversi motivi. Ha, come Centro di Riferimento, I'Azienda Ospedaliera "Card. G. Panico" di Tricase in provincia di Lecce e fornisce 26 posti "rene" che sono attivi 12 ore (18 durante il periodo estivo). Nel nostro Centro la tecnica BH è stata iniziata nel Maggio del 2011, in seguito alla formazione del personale infermieristico e medico e alla sensibilizzazione dei pazienti che erano stati individuati per l'utilizzo di tale metodica. II ricorso a questa nuova modalità di puntura, affiancata alle altre tecniche, non come alternativa ma come una procedura diversa che limitasse le complicazioni a breve e a lungo termine, è stato motivato dalla lettura di alcuni studi in merito all'argomento, i quali sembravano dimostrare un alto gradimento da parte del personale e dei pazienti e un'efficacia clinica in termini di benefici $(6,7)$. Tale tecnica deve essere affidata a personale esperto e motivato, considerando che è impegnativo creare un adeguato sito di puntura e che la manualità con gli aghi smussi viene acquisita con la pratica e la costanza (12) (quindi una sfida per tutto il team ad allargare le competenze e ad applicare le best practices). La scelta dei pazienti è stata effettuata collegialmente, tenendo conto di alcuni criteri quali: la difficoltà di puntura, l'eccessivo sanguinamento post dialisi e la prevenzione di formazioni trombotiche e/o aneurismatiche o di tessuti cicatriziali "antiestetici", soprattutto nei soggetti giovani (dopotutto, come affermato da Pegoraro, le persone hanno bisogno di "sentirsi meno malate, meno brutte e meno fragili" (13). È stato adottato il protocollo di puntura a sito costante proposto da EDTNA-ERCA (14) ed ogni paziente è stato affidato a 2 infermieri che dovevano collaborare insieme nella scelta dei siti e nel mantenere la stessa inclinazione e la stessa direzione nell'inserzione degli aghi. Questo ha comportato delle difficoltà organizzative, che vengono percepite ancora oggi, poiché non è sempre auspicabile far coincidere i turni dei pazienti con quelli degli infermieri 
durante il periodo di preparazione dei siti. Nonostante ciò, negli anni seguenti, la tecnica è stata estesa a 24 pazienti (su 60 , di cui il $62.5 \%$ con FAV nativa) in emodialisi periodica (19 maschi e 5 femmine) con età media 57 anni (range 20-75) e anzianità dialitica media pari a 41 mesi (range 5 mesi-24 anni). Questo ha permesso, soprattutto agli infermieri, di dedicare più attenzione alla tecnica, di perfezionarla e di acquisire una "buona pratica clinica", riuscendo a individuare per tempo le criticità e a superarle con abilità, spirito di autoverifica e collaborazione, con la condivisione di scelte e strategie. In questi pazienti si sono verificati: un fallimento, probabilmente legato a peculiarità anatomiche che non hanno permesso un efficiente allineamento tra il sito cutaneo e la parete vasale ("venus flap") (15), un episodio di trombosi dell'AV, che ha comportato l'inserzione di un CVC in giugulare nell'attesa di risolvere il problema, un episodio di infezione localizzata, che è stata curata con mupirocina topica, e un episodio di sepsi da Staphylococcus Aureus, di dubbia provenienza, dato che la paziente, per un breve periodo, è stata portatrice di un CVC. Quest'ultima è stata trattata con terapia antibiotica per via sistemica ed è guarita in poche settimane, durante le quali si è continuato a pungere negli stessi siti $\mathrm{BH}$ (per una difficoltà oggettiva nel reperire altri vasi), incrementando la sorveglianza sanitaria e trattando ogni occhiello come se fosse il sito di un CVC. Questi episodi hanno fatto in modo che venisse rivisto il protocollo per la disinfezione della FAV, sensibilizzando sia il personale nell'osservare scrupolosamente le procedure sia i pazienti nell'avere maggiore cura del proprio AV. Oggi la paziente è guarita completamente ed è anche riuscita ad autopungersi sotto tutoraggio dell'operatore di sala.

La procedura per la preparazione della FAV con incannulazione Buttonhole utilizzata nella nostra U.O. di dialisi è citata nella Tabella I.

\section{Caso clinico}

Descriviamo la storia clinica di un uomo di 49 anni, in emodialisi periodica dall'età di 25 , in seguito a glomerulonefrite cronica mesangioproliferativa. Il primo accesso vascolare utilizzato fu una fistola arterovenosa distale all'avambraccio sinistro. Dopo 2 anni di trattamento dialitico, il paziente venne sottoposto a trapianto di rene da donatore cadavere. In seguito a rigetto cronico, dopo poco più di 2 anni, riprese il trattamento sostitutivo, utilizzando un Catetere Venoso Centrale (CVC) in vena giugulare destra, non potendo utilizzare la FAV per una trombosi secondaria a stenosi. Successivamente venne sottoposto all'allestimento di una nuova FAV, poco più a monte rispetto alla precedente, e, per la venipuntura, venne adottata la tecnica a scala di corda. Tale FAV ha avuto uno sviluppo ottimale, di facile pungibilità e di buona portata ed efficienza dialitica e venne utilizzata per circa 19 anni senza problemi rilevanti, anche se, con il passare degli anni, si crearono delle dilatazioni aneurismatiche di vari segmenti, sia all'avambraccio che al braccio, come conseguenza delle ripetute e reiterate punture (Fig. 1). A Febbraio 2014 si sono verificati, in corrispondenza delle dilatazioni aneurismatiche, episodi di infezioni cutanee con arrossamento della cute, che è diventata traslucida, più sottile e più tesa. Una valutazione ecocolordoppler della FAV (Fig. 2) ha evidenziato, all'interno delle sacche aneurismatiche, la presenza di materiale ipoisoecogeno da riferire a trombi. Gli episodi infettivi associati alle trombosi delle sacche aneurismatiche si sono ripresentati, tanto da rendere necessaria la chiusura dell'accesso vascolare. Permangono tuttora le dilatazioni aneurismatiche, non ancora corrette chirurgicamente, perché comportano un intervento impegnativo. Nel frattempo, è stata allestita una nuova fistola all'avambraccio destro (Fig. 3) ed è stato posizionato un CVC temporaneo in vena giugulare destra in attesa della maturazione della FAV. Per la puntura del nuovo AV, con-

TABELLA I - Protocollo di puntura utilizzato nella U.O. di Nefrologia e Dialisi dell’Ospedale di Tricase

- Educare il paziente a eseguire il lavaggio del braccio della FAV con acqua e sapone prima della seduta

- Frizionare le mani dell'operatore prima e dopo contatto con il paziente con clorexidina alcolica

- Indossare guanti puliti e mascherina coprifaccia

- Far indossare al paziente una mascherina coprifaccia e guanti in caso di auto-puntura

- Effettuare antisepsi della superficie cutanea del braccio applicando clorexidina al $2 \%$ in soluzione alcolica (3 min) o, in caso di ipersensibilità o allergia, iodo povidone al $10 \%$ (per 2 o 3 min)

- $\quad$ Rimuovere le croste servendosi di pinze appuntite sterili o degli appositi dispositivi forniti dai produttori di aghi smussi

- $\quad$ Ripetere antisepsi dopo la rimozione delle croste

- $\quad$ Inserire gli aghi evitando possibili contaminazioni degli stessi

Al termine della seduta

- $\quad$ Operatore e paziente devono indossare mascherina coprifaccia e guanti puliti

- Eseguire il disinserimento degli aghi servendosi di garze sterili previa antisepsi con iodopovidone spray dei siti prima di estrarre gli aghi

- Non disinserire gli aghi con i guanti dedicati per lo stacco e altre manovre tipo smontaggio del monitor etc. e provvedere alla sostituzione degli stessi. 


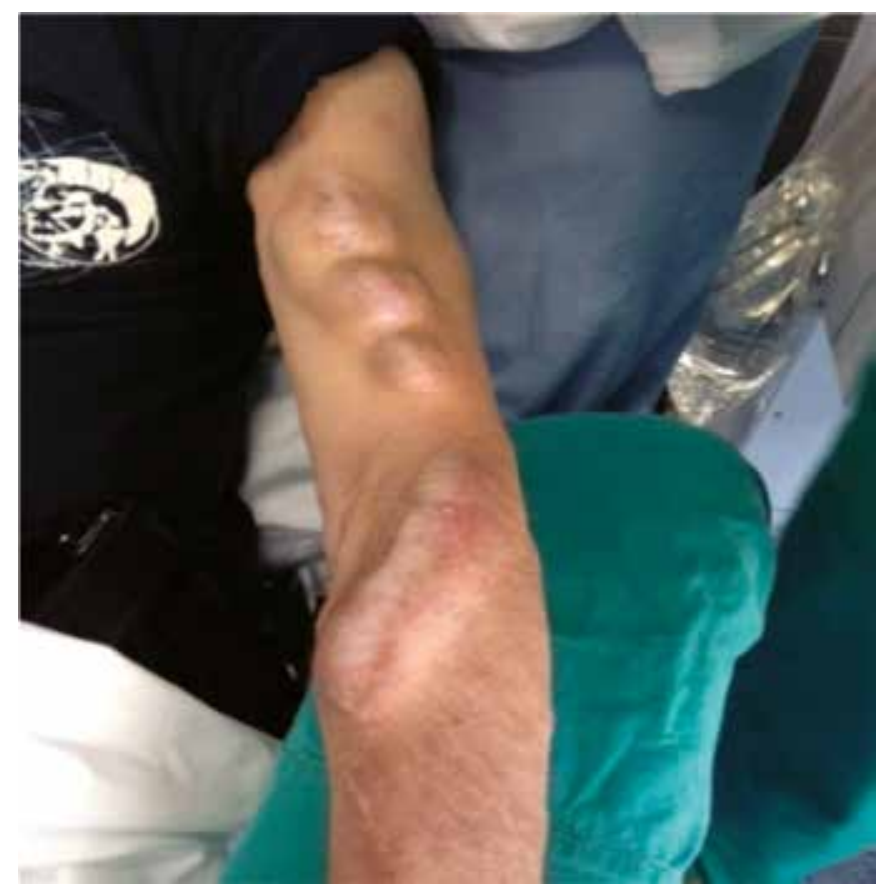

Fig. 1 - Dilatazioni aneurismatiche conseguenti alle ripetute punture della FAV con tecnica RL.

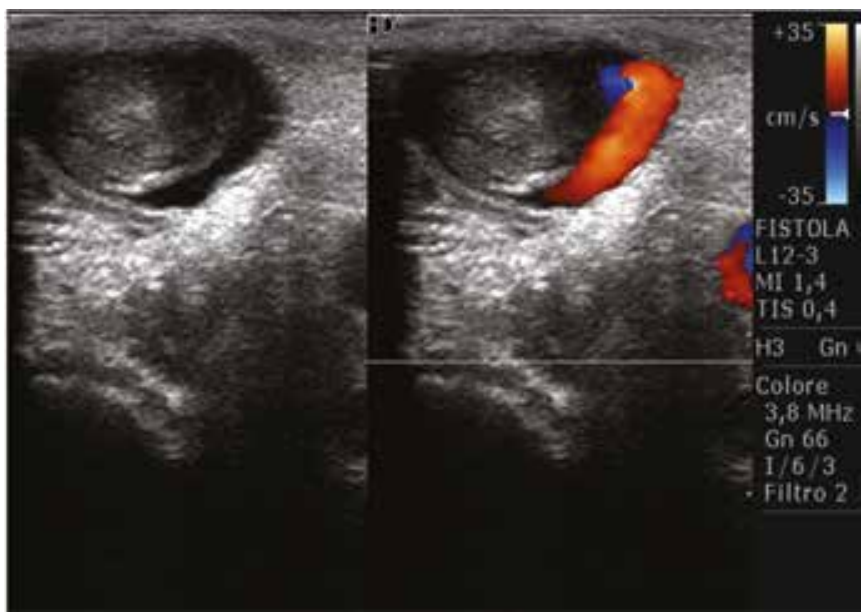

Fig. 2 - Ecocolordoppler FAV: presenza di formazione trombotica in sede aneurismatica.

siderando i problemi avuti con la metodica precedente, si è deciso di ricorrere alla tecnica $\mathrm{BH}$ con il consenso del paziente, al quale sono stati spiegati i possibili vantaggi e svantaggi della stessa. Una volta maturata la FAV sono stati individuati $\mathrm{i}$ siti di inserzione degli aghi, a una distanza di circa $8 \mathrm{~cm}$ l'uno dall'altro. Per l'allestimento degli occhielli sono stati scelti 2 infermieri esperti e motivati, i quali hanno utilizzato aghi taglienti (15 G) per 8-9 sedute, periodo in cui sono stati utilizzati, a fine seduta, i dispositivi "biohole ${ }^{T M}$ " (6), che sono stati

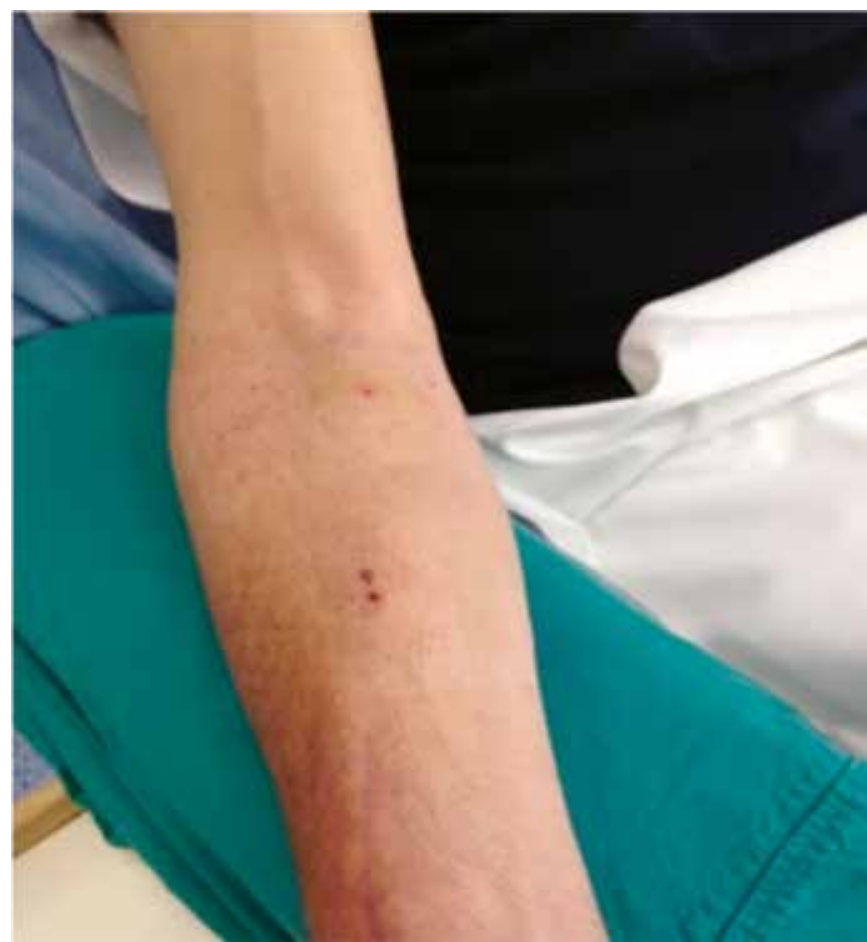

Fig. 3 - Braccio nuova FAV.

molto utili nel mantenere pervio il tragitto sottocutaneo fino al vaso. Successivamente, si è passati all'inserimento degli aghi smussi, che ha avuto successo fino a quando non hanno cominciato a pungere anche gli altri infermieri i quali, nonostante seguissero le indicazioni dei colleghi referenti, alcune volte riscontravano difficoltà nel reperire entrambi i vasi. Decisivo è stato il coinvolgimento del paziente, il quale, anche se non ha avuto il coraggio di effettuare l'auto-puntura, ha comunque dato un importante contributo nell'aiutare l'infermiere durante la venipuntura, indicando la direzione e l'angolazione nell'inserzione dell'ago e comunicando le proprie sensazioni e riconoscendo precocemente eventuali problemi. A distanza di 16 mesi, il paziente riferisce benefici in termini di riduzione del dolore da puntura e del tempo di emostasi e di presenza di tessuto cicatriziale meno visibile (Fig. 4). L'unico esito negativo, riferito anche da altri pazienti, sembra essere il dolore avvertito durante la rimozione delle croste dai siti di inserzione degli aghi.

\section{Discussione}

Alcuni studi hanno dimostrato che l'uso della tecnica a sito costante $(\mathrm{BH})$ riduce le complicazioni associate alla puntura $(3,5,7,8,16,17)$, mentre altri studi hanno messo in evidenza il ricorso all'uso di antibiotici per curare alcune infezioni accesso-sito-correlate $(8,18-22)$, in particolare quelle da Staphilococcus Aureus, che è la causa di più dell' $8 \%$ di mortalità nella popolazione dialitica $(8,23)$. Comunque, 


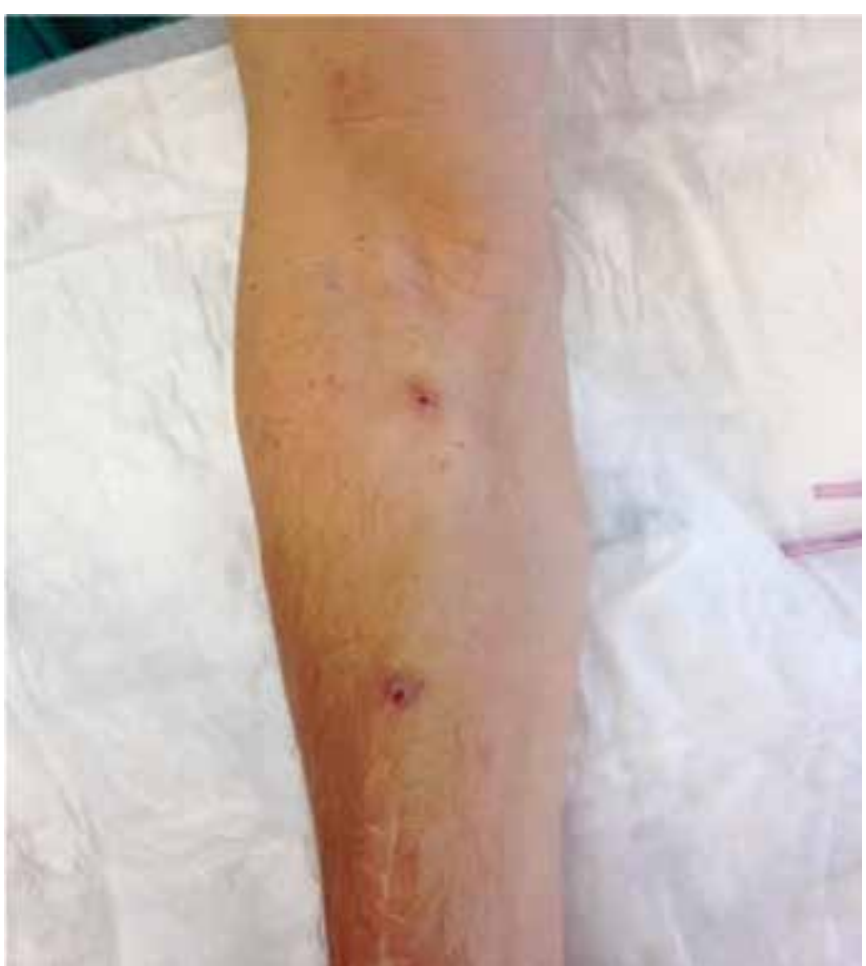

Fig. 4 - FAV punta con tecnica BH a distanza di 16 mesi.

la tecnica $\mathrm{BH}$ sembra essere quella preferita sia dai pazienti che dal personale medico e infermieristico per la facilità di puntura, per la minore incidenza di ematomi, per la riduzione del dolore e del tempo di emostasi post dialitico e per la formazione, in sede di puntura, di un tessuto cicatriziale meno visibile (24). Uno studio randomizzato, condotto su 70 pazienti, ha messo in evidenza, nel gruppo $\mathrm{BH}$, oltre a un maggior numero di infezioni e di ematomi, una maggiore percezione del dolore (25). Per contro, altri lavori rilevano una diminuzione della percezione del dolore da parte dei pazienti punti con la tecnica $\mathrm{BH}$, tanto da preferire gli aghi smussi a quelli taglienti $(26,27)$. Secondo Napoli, il tunnel creato dalla tecnica $\mathrm{BH}$, rispetto ai tunnel dei Cateteri per Dialisi Peritoneale (CP) e dei Cateteri Venosi Centrali (CVC), sembrerebbe più esposto alla colonizzazione batterica, perché senza la cuffia di dacron, che ha proprio il fine di esercitare una barriera protettiva contro l'ingresso dei germi (28). Un articolo, pubblicato di recente, riporta il caso di un paziente in trattamento emodialitico periodico sottoposto a venipuntura con tecnica $\mathrm{BH}$, sul quale è stata riscontrata una disfunzione dell'AV con un ricircolo dell' $87 \%$. Un'ecografia vascolare ha rivelato la presenza di una fistola anomala tra I'AV e la vena e la presenza di un trombo con dilatazione aneurismatica nei pressi dell'anastomosi (29). Complessivamente, i dati derivanti dalla letteratura riportano risultati contrastanti che hanno creato (e creano) non poche perplessità e discussioni nella comunità scientifica di interesse. C'è da chiedersi: come mai una tecnica che adotta gli stessi protocolli porta a esiti diversi? Quali errori vengono commessi? II dato certo è che la tecnica $\mathrm{BH}$ è una Pratica Avanzata che richiede la messa in atto di strategie organizzative attente e la presenza di figure di riferimento clinico e gestionale con Competenze Avanzate. Galli et al. affermano che la tecnica $\mathrm{BH}$ richiede "...un vero e proprio cambiamento culturale che dovrebbe riguardare non solo il personale ma anche la Dirigenza Medico-Infermieristica, la Direzione Sanitaria e l'Organizzazione Sindacale... e richiedere... un numero limitato di pungitori in grado di praticare la puntura non solo con l'ago tagliente ma anche con l'ago smusso... (30). L'accesso vascolare e il percorso di competenza specialistica avanzata sono considerati fondamentali (core competence) e non certo residuali all'interno di un Centro Dialisi. Come sottolineato da Lombardi e Quintaliani: "all'interno di conoscenze, skill e attitudini, bisogna dapprima conoscere, poi conoscere come, poi dimostrare di saper fare e, da ultimo, fare" (12).

\section{Conclusioni}

Da quanto emerso dalla letteratura nei confronti della tecnica $\mathrm{BH}$, ai fini della salvaguardia dell'accesso vascolare, sembra sia necessario riservare un'attenzione particolare a diversi aspetti:

1. la formazione (re-training) del personale addetto alla venipuntura;

2. l'empowerment dei pazienti;

3. la corretta preparazione dei siti per la puntura;

4. il rispetto rigoroso di procedure mirate alla prevenzione della sepsi;

5. la sorveglianza (test di ricircolo, ecocolordoppler, ecc.) dell'accesso vascolare prima e nel corso dell'utilizzo della tecnica.

In base alla nostra esperienza, la tecnica $\mathrm{BH}$, affiancata alle altre modalità di puntura della FAV nativa del paziente emodializzato cronico, può essere "perfettibile" (31) se vengono mantenuti un buon clima organizzativo e un alto livello di motivazione e di attenzione, rispettando rigidi protocolli di antisepsi e garantendo una formazione adeguata e aggiornata al personale sanitario addetto alla venipuntura (definizione di figure di Competenza Avanzata (13)), il quale assume un ruolo fondamentale sia nell'esecuzione corretta delle manovre sia nell'educazione e nel coinvolgimento del paziente (empowerment). Sono, comunque, necessari ulteriori studi su una popolazione più ampia e con follow-up più lunghi per confermare l'utilità del metodo in termini di efficienza e di efficacia.

\section{Ringraziamenti}

Un ringraziamento speciale va alla Dr.ssa Anna Mudoni per la sua preziosa collaborazione. 


\section{Disclosures}

Financial support: No financial support was received for this submission.

Conflict of interest: The authors have no conflict of interest.

\section{Bibliografia}

1. Maya ID, Allon M. Vascular access: core curriculum 2008. Am J Kidney Dis 2008;51(4):702-8.

2. Ball LK. Improving arteriovenous fistula cannulation skills. Nephrol Nurs J 2005;32:611-7.

3. Krönung G. Plastic deformation of Cimino fistula by repeated puncture. Nephrol Dial Transplant 1984;635-8.

4. Twardowski ZJ. The Buttonhole Method Spreads "Like Wildfire". Dialysis \& Transplantation 2011;40:443.

5. Twardowski ZJ. Constant site (Buttonhole) method of needle insertion for hemodialysis. Dial Transplant 1995;24:559-76.

6. Toma S, Shinzato T, Fukui $H$, et al. A timesaving method to create a fixed puncture route for the buttonhole technique. Nephrol Dial Transplant 2003;18(10):2118-21.

7. Twardowski ZJ, Kubara H. Different sites versus constant site of needle insertion into arteriovenous fistulas for treatment by repeated dialysis. Dialysis \& Transplant 1979;8:978-80.

8. van Loon MM, Goovaerts T, Kessels AG, van der Sande FM, Tordoir JH. Buttonhole needling of haemodialysis arteriovenous fistulae results in less complications and interventions compared to the rope-ladder technique. Nephrol Dial Transplant 2010;25:225-30.

9. NFK-KDOQKI. Clinical practice guidelines and clinical practice recommendations for vascular access: update 2006. Am J Kidney Dis 2006;48(Suppl. 1):S176-322.

10. Mott $S$. The art of teaching buttonhole self-cannulation. Home Dialysis Central 2011; http://homedialysis.org/buttonhole.

11. Verhallen AM, Kooistra MT, van Jaarsveld BC. Cannulating in hemodialysis: rope-ladder or buttonhole technique? Nephrol Dial Transplant 2007;22:2601-4.

12. Lombardi M, Quintaliani G. Quale operatore, per quale venipuntura, per quale paziente in emodialisi? Giornale di Tecniche Nefrologiche e Dialitiche 2014;26(1):1-3.

13. Pegoraro M. Puntura a Occhiello: cosa c'è di nuovo? Giornale di Tecniche Nefrologiche e Dialitiche 2013;25(2):92-3.

14. Pegoraro M. La puntura a occhiello della FAV arterovenosa. Storia e dati di evidenza nell'applicazione della tecnica. Giornale di Tecniche Nefrologiche e Dialitiche 2008;20(1-2):1-6.

15. Pegoraro M. Puntura a Occhiello: risposte alle domande più frequenti. Giornale di Tecniche Nefrologiche e Dialitiche 2014; 26(4):368-73.
16. Ball L, Treat $L$, Riffle $V$, et al. A multi-center perspective of the buttonhole technique in the Pacific Northwest. Nephrol Nurse J 2007;34:234-41.

17. Twardowski ZJ. Buttonhole method for needle insertion into A-V fistula. Nefrologia i Dializoterapia Polska 2006;10:156-8.

18. Verhallen A. Successful vascular access management for home haemodialysis: a practical approach. J Ren Care 2013;39(1): 28-34.

19. Leon C, Orozco-Vargas LC, Krishnamurthy G, et al. Accuracy of physical examination in the detection of arteriovenous grafts stenosis. Semin Dial 2008;21:85-8.

20. Marticorena RM, Hunter J, Macleod S, et al. The salvage of aneurismal fistulae utilizing a modified buttonhole cannulation. Hemodial Int 2006;10:193-200.

21. Doss S, Schiller B, Moran J. Buttonhole cannulation--an un expected outcome. Nephrol Nurs J 2008;35:417-9.

22. Silva Gdos S, Silva RA, Nicolino AM, et al. Initial experience with the buttonhole technique in a Brazilian hemodialysis center. J Bras Nefrol 2010;32(3):256-60.

23. Vandecasteele SJ, Boelaert JR, De Vriese AS. Staphylococcus aureus infections in hemodialysis: what a nephrologist should know. Clin J Am Soc Nephrol 2009;4:1388-400.

24. Leitch R, Ouwendyk M, Freguson E. Nursing issues related to patient selection, vascular access, and education quotidian hemodialysis. Am J Kidney Dis 2003;42:S56-60.

25. Chow J, Rayment G, San Miguel S, Gilbert M. A randomised controlled trial of buttonhole cannulation for the prevention of fistula access complications. J Ren Care 2011;37(2):85-93.

26. Figueiredo $A E$, Viegas $A$, Monteiro $M$, et al. Research into pain perception with arteriovenous fistula (AVF) cannulation. J Ren Care 2008;34(4):169-72.

27. Hashmi A, Cheema MQ, Moss AH. Hemodialysis patients' experience with and attitudes toward the buttonhole technique for arteriovenous fistula cannulation. Clin Nephrol 2010;74(5): 346-50.

28. Napoli M. II buttonhole: come trasformare una fistola in un catetere. Giornale di Tecniche Nefrologiche e Dialitiche 2013;25(4):290-2.

29. Wen Cao, Lin Chen, Mei Shi, Li Zhou, Ping Fu. Formation of abnormal Fistula on Arteriovenous Fistula in a Patient with Buttonhole Cannulation: A Case Report. Case Rep Nephrol Dial 2015;5(1):71-6.

30. Galli S, Zollo A, Cavatorta F. La puntura a occhiello della fistola artero-venosa: pro e contro. Giornale di Tecniche Nefrologiche e Dialitiche 2014;26(1):8-11.

31. Lombardi M. La tecnica di puntura a sito costante (Buttonhole technique). Giornale di Tecniche Nefrologiche e Dialitiche 2013;25(4):289. 